\title{
Sex differences in traumatic stress reactivity in rats with and without a history of alcohol drinking
}

\author{
Lucas Albrechet-Souza ${ }^{1,2^{*}}$, Connor L. Schratz ${ }^{1}$ and Nicholas W. Gilpin ${ }^{1,2,3,4}$
}

\begin{abstract}
Background: Alcohol misuse and post-traumatic stress disorder (PTSD) are highly comorbid, and treatment outcomes are worse in individuals with both conditions. Although more men report experiencing traumatic events than women, the lifetime prevalence of PTSD is twice as high in females. Despite these data trends in humans, preclinical studies of traumatic stress reactivity have been performed almost exclusively in male animals.

Methods: This study was designed to examine sex differences in traumatic stress reactivity in alcohol-naive rats (experiment 1) and rats given intermittent access to 20\% ethanol in a 2-bottle choice paradigm for 5 weeks (experiment 2). Animals were exposed to predator odor (bobcat urine) and tested for contextual avoidance $24 \mathrm{~h}$ later; unstressed controls were never exposed to predator odor. We evaluated changes in physiological arousal using the acoustic startle response (ASR) test at day 2 post-stress and anxiety-like behavior measured in the elevated plus-maze (EPM) at day 17 post-stress. In experiment 3, time course of corticosterone response was examined in male and female rats following exposure to predator odor stress.
\end{abstract}

Results: Alcohol-naive males and females exposed to predator odor displayed blunted weight gain $24 \mathrm{~h}$ post-stress, but only a subset of stressed animals exhibited avoidance behavior. In alcohol-drinking animals, the proportion of avoiders was higher in males than females, and predator odor exposure increased ASR in males only. Stressed females exhibited blunted ASR relative to unstressed females and stressed males, regardless of alcohol drinking history. Alcohol-experienced females presented lower anxiety-like behavior and higher general activity in the EPM in comparison with alcohol-experienced males. Plasma corticosterone levels were higher in females immediately after predator odor exposure until 60 min post-stress relative to males.

Conclusions: We report robust sex differences in behavioral and endocrine responses to bobcat urine exposure in adult Wistar rats. Also, males with a history of chronic moderate alcohol drinking exhibited increased traumatic stress reactivity relative to alcohol-drinking females. Our findings emphasize the importance of considering sex as a biological variable in the investigation of traumatic stress effects on physiology and behavior.

Keywords: Sex differences, Predator odor, Bobcat urine, Alcohol, Stress, Trauma, Startle, Arousal, Corticosterone

\footnotetext{
* Correspondence: Idesou@|suhsc.edu

'Department of Physiology, School of Medicine, Louisiana State University Health Sciences Center, New Orleans, LA, USA

${ }^{2}$ Alcohol \& Drug Center of Excellence, School of Medicine, Louisiana State

University Health Sciences Center, New Orleans, LA, USA

Full list of author information is available at the end of the article
}

(c) The Author(s). 2020 Open Access This article is licensed under a Creative Commons Attribution 4.0 International License, which permits use, sharing, adaptation, distribution and reproduction in any medium or format, as long as you give appropriate credit to the original author(s) and the source, provide a link to the Creative Commons licence, and indicate if changes were made. The images or other third party material in this article are included in the article's Creative Commons licence, unless indicated otherwise in a credit line to the material. If material is not included in the article's Creative Commons licence and your intended use is not permitted by statutory regulation or exceeds the permitted use, you will need to obtain permission directly from the copyright holder. To view a copy of this licence, visit http://creativecommons.org/licenses/by/4.0/ The Creative Commons Public Domain Dedication waiver (http://creativecommons.org/publicdomain/zero/1.0/) applies to the data made available in this article, unless otherwise stated in a credit line to the data. 


\section{Background}

Post-traumatic stress disorder (PTSD) is a chronic psychiatric disease that is seen in some but not all individuals after experiencing a traumatic event. Major diagnostic criteria for PTSD include re-experiencing the traumatic event, negative affective state, exaggerated startle responses, and persistent avoidance of traumarelated cues [1]. Women are twice as likely to develop PTSD after trauma [2, 3], and women with trauma exposure and/or PTSD exhibit more sensitivity to and less tolerance of negative emotions $[4,5]$.

Alcohol use disorder (AUD) is one of the most common co-occurring conditions among individuals diagnosed with PTSD [6, 7]. Approximately one third of individuals with lifetime PTSD also meet the criteria for AUD [8]. Some populations, such as military personnel, are at high risk for AUD and PTSD comorbidities. For example, in Iraq and Afghanistan veterans, 63\% of those diagnosed with AUD also met the criteria for PTSD [9]. Although men have a higher prevalence of AUD than women, and women have a higher prevalence of PTSD than men, any individual with either disorder is more likely to have the other [10].

Exaggerated startle response is considered a hallmark symptom of PTSD [1] and is predictive of disease severity in combat-exposed veterans returning home from active duty [11]. In a sample of predominantly male (76.8\%) military service members referred for psychiatric evaluation for suicide-related concerns, the hyperarousal symptom cluster was the only significant predictor of subsequent suicide attempts [12]. Although elevated autonomic responses to startling tones have been reported in male and female trauma survivors [13-15], investigations of startle responsivity in patients with PTSD have produced mix results. Male Gulf War veterans with PTSD exhibited exaggerated startle compared to nonPTSD veterans [16]. Conversely, male Vietnam veterans with PTSD did not show increased startle [17] unless they were subjected to a stressful environment [18]. Interestingly, studies in either all [19] or mostly female (66.7\%) humans with PTSD [20] report blunted motor responses to acoustic stimuli, suggesting a potential sex difference in traumatic stress effects on acoustic startle responding.

Preclinical studies using animal models to recapitulate PTSD-like behavioral deficits allow investigation of the biological mechanisms underlying traumatic stress effects, but most preclinical research has been conducted in male animals, potentially neglecting issues specific to female subjects $[21,22]$. Predator exposure and predator scent are ethologically relevant stressors commonly used as animal models of PTSD. Rodents are exposed to predator odor (PO) in a variety of ways, including cloths containing odor (cat), urine (bobcat, fox), feces or litter, or trimethylthiazoline (TMT) - a synthetic compound isolated from fox feces [23-26]. Although predator stress has been shown to elicit lasting increases in freezing and avoidance behaviors [27, 28], the effects of PO stress on acoustic startle response (ASR) are not consistent. For example, male rats exhibit potentiated ASR magnitude during exposure to TMT [29], but three exposures to cat odor (10 min per day separated by a 48 -h inter-exposure interval) failed to promote persistent changes on startle reactivity [30].

The current experiments were designed to explore sex differences in traumatic stress reactivity in rats exposed to a PO stress model using bobcat urine developed in our laboratory [31]. The urine of carnivorous species contains 2-phenylethylamine, a trace amine produced by the breakdown of the amino acid phenylalanine [32] that activates trace amine-associated receptor 4 in the rodent olfactory cortex and produces avoidance behavior in rodents [32]. In experiment 1 , we tested how male and female rats respond to bobcat urine exposure on a variety of behavioral paradigms including context avoidance, acoustic startle, and the elevated plus-maze (EPM) test. In experiment 2, we evaluated whether stress responsivity to PO changes following chronic voluntary alcohol consumption. Finally, in experiment 3 , we examined sex effects on the time course of corticosterone response following exposure to bobcat urine in alcohol-naive male and female rats.

\section{Methods}

\section{Animals}

Eight-week-old male and female Wistar rats (Charles River, Raleigh, NC) were housed in same-sex pairs in a humidity- and temperature-controlled $\left(22{ }^{\circ} \mathrm{C}\right)$ vivarium on a 12-h reversed light-dark cycle (lights off at 7 AM). Animals had ad libitum access to food and water throughout the experiments and were handled daily for 3 min for 1 week before the initiation of experimental protocols. Female rats were freely cycling and assigned to treatment groups without regard to estrous cycle stage.

\section{Study design}

In experiment 1 , rats were exposed to bobcat urine and tested for avoidance of the odor-paired context $24 \mathrm{~h}$ later ( $n=24$ males and 24 females); unstressed controls were never exposed to PO ( $n=8$ males and 8 females). ASR was assessed at day 2 post-stress, and anxiety-like behavior was evaluated at day 17 post-stress using the EPM (Fig. 1a). In experiment 2, pair-housed rats were given intermittent access to $20 \%$ ethanol in a 2-bottle choice paradigm for 5 weeks before exposure to bobcat urine. Rats underwent the behavioral test battery as described in experiment 1 (Fig. 2a). This experiment involved 4 
a

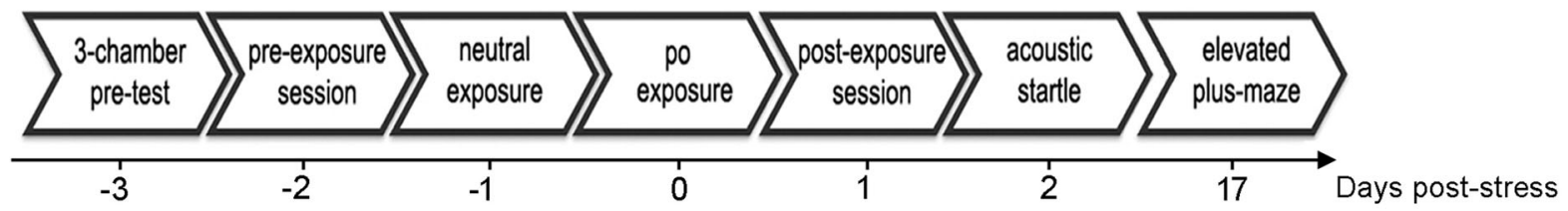

\section{b $\Delta$ Time in PO-Paired Context}

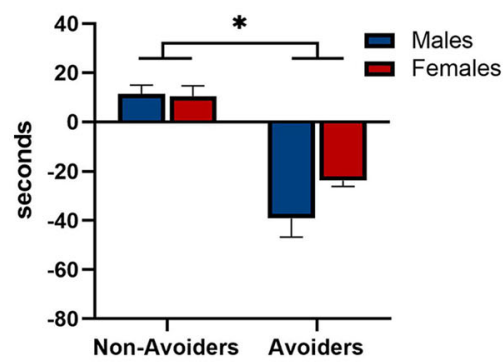

e Acoustic Startle $(95 \mathrm{~dB})$

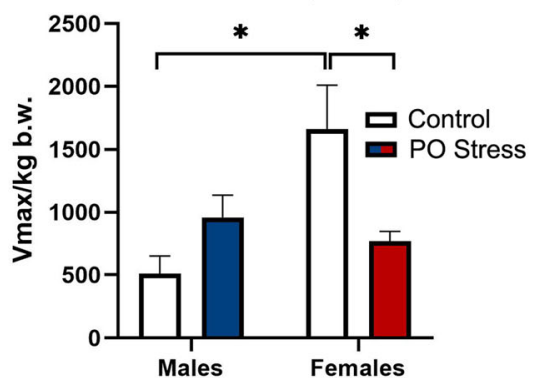

C

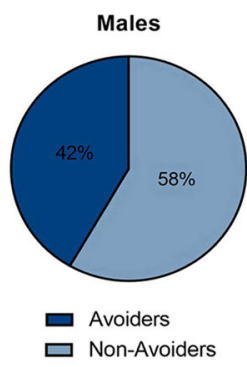

f

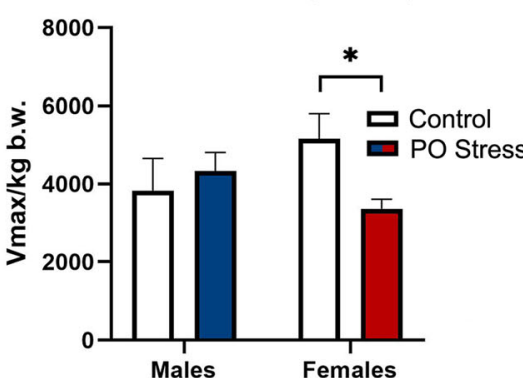

d

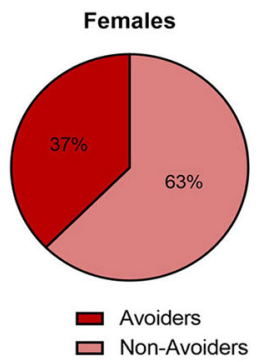

Weight Gain 24h Post-Stress

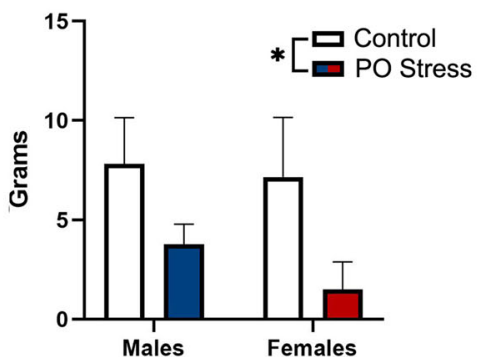

g Acoustic Startle $(115 \mathrm{~dB})$

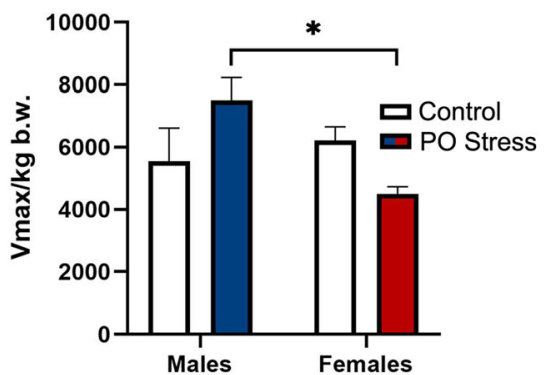

Fig. 1 Traumatic stress response in alcohol-naive male and female rats. a Experimental design. Rats underwent the conditioned place aversion paradigm using bobcat urine. Controls were never exposed to predator odor (PO) stress. Avoidance behavior was measured $24 \mathrm{~h}$ post-stress (day 1). Acoustic startle reactivity was evaluated at day 2 post-stress and is expressed as Vmax normalized by body weight in kilograms (Vmax/kg b.w.). Anxiety-like behavior was tested at day 17 after PO exposure. $\mathbf{b}$ Change in time spent in PO-paired chamber in rats indexed as avoiders or nonavoiders. c Avoidance distribution of stressed rats (avoiders $\times$ non-avoiders). $\mathbf{d}$ Weight gain measured $24 \mathrm{~h}$ after exposure to PO. e Acoustic startle response at $95 \mathrm{~dB}$. f Acoustic startle response at $105 \mathrm{~dB}$. g Acoustic startle response at $115 \mathrm{~dB}$. Data presented as mean $\pm \mathrm{SEM}$. Asterisk denotes $P$ $<.05$

groups: control males $(n=6)$, control females $(n=6)$, stressed males $(n=12)$, and stressed females $(n=10)$. In experiment 3 ( $n=6$ males and 6 females), rats were exposed to bobcat urine in a clean cage. Tail blood samples for corticosterone analyses were collected before exposure to PO, immediately after, $30 \mathrm{~min}, 60 \mathrm{~min}$, and $90 \mathrm{~min}$ post-stress (Fig. 3a). These experiments were conducted with independent cohort of rats. Animals were tested in the morning between 9:00 AM and 12:00 PM. At the end of the experiments, rats were euthanized by decapitation under isoflurane anesthesia.

\section{Predator odor stress}

Rats were tested in a 5-day conditioned place aversion procedure [31] that began after the acclimatization period in alcohol-naive rats (experiment 1) or $24 \mathrm{~h}$ after the last drinking session in alcohol-experienced rats (experiment 2). On the first day (day -3, Figs. 1a and 2a), rats were allowed 5 min of free exploration of the apparatus (3-chamber pre-test session), which consisted of three large chambers $(36 \mathrm{~cm}$ length $\times 30 \mathrm{~cm}$ width $\times 34$ $\mathrm{cm}$ height) with different types of floor texture (circles, grid, or rod floor) and patterned walls (circles, white, or stripes), separated by a small triangular connecting chamber. The apparatus was thoroughly cleaned between animals with Quatricide ${ }^{\circledast} \mathrm{PV}$ in water at a concentration of 1:64 (Pharmacal Research Labs, Waterbury, CT). For each rat, the chamber that exhibited the most deviant time score of the three (i.e., highly preferred or highly avoided) was excluded from all future sessions for that rat. On the next day (day -2), the rat was allowed 5 min to explore the two non-excluded conditioning chambers (pre-exposure session). Rats were assigned to PO stress or unstressed control groups that were counterbalanced for the magnitude of baseline preference for one chamber versus the other (i.e., groups were assigned 


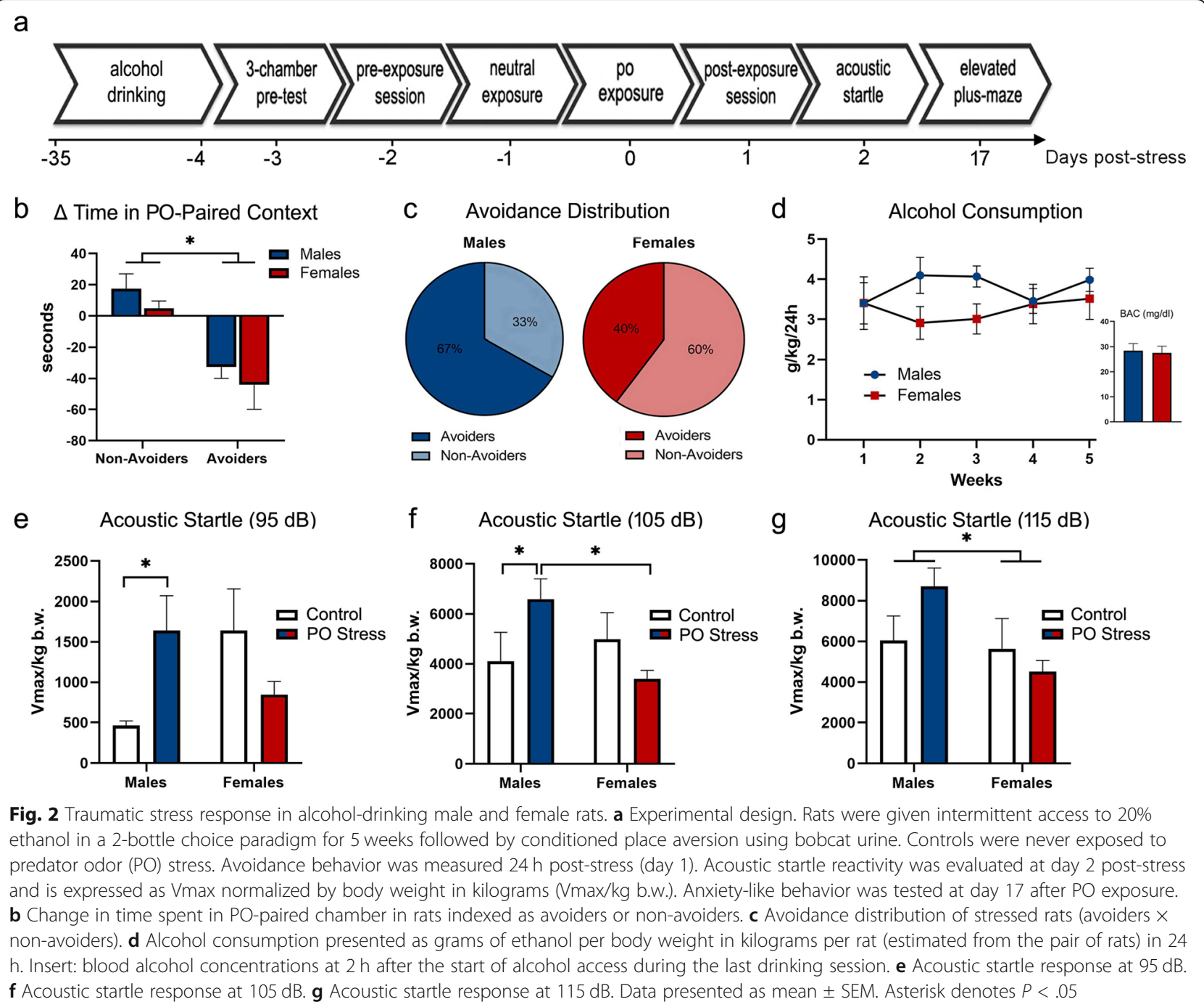

such that mean pre-existing preference for each of the two chambers was approximately zero for PO stress and control groups). For rats in the PO stress group, an unbiased and counterbalanced design was used to determine which chamber (i.e., more preferred or less preferred) would be paired with PO for each rat. On the next day (day -1), each rat was placed in one of the two chambers with the guillotine door shut without odor for $15 \mathrm{~min}$ (neutral exposure). On day 0 , rats were placed in the other chamber with the guillotine door shut and a sponge soaked with $3 \mathrm{ml}$ of bobcat urine (Lynx rufus; Maine Outdoor Solutions, Hermon, ME) placed under the floor for $15 \mathrm{~min}$ (odor exposure). Control rats were treated identically to PO-exposed rats, but the sponges did not contain bobcat urine. On day 1 , rats were allowed to explore the two chambers for $5 \mathrm{~min}$ (post-exposure session). All testing was conducted under indirect dim illumination (one $60 \mathrm{~W}$ white light facing the wall providing approximately $10 \mathrm{~lx}$ in the apparatus), and all sessions were recorded and time spent in each chamber was scored by a treatment-blind observer. Avoidance was quantified as a difference score calculated as time spent in odor-paired chamber on day 1 minus time spent in the same chamber on day -2 . Rats that displayed $>10-\mathrm{s}$ decrease in time spent in the PO-paired context were classified as avoiders; all other bobcat urine-exposed rats were classified as non-avoiders.

\section{Intermittent access 2-bottle choice alcohol homecage drinking}

Pair-housed adult male and female Wistar rats were given intermittent access to alcohol and water in three 24-h sessions per week for 5 weeks prior to PO stress [33]. We chose this housing condition to avoid exposing animals to social isolation stress. Briefly, rats were 
a

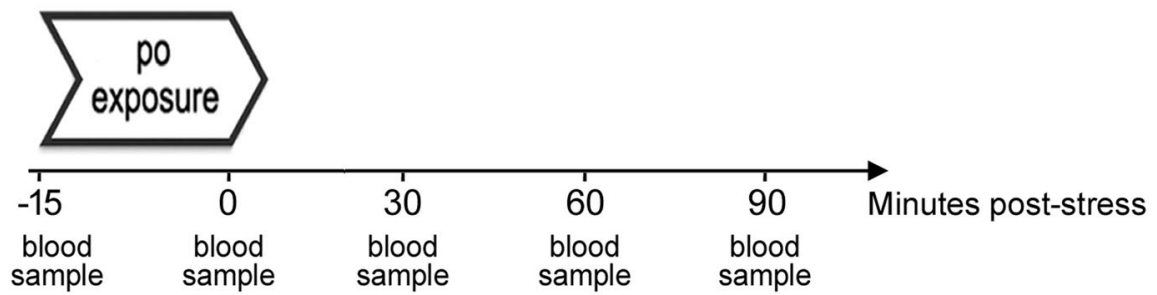

b Plasma Corticosterone

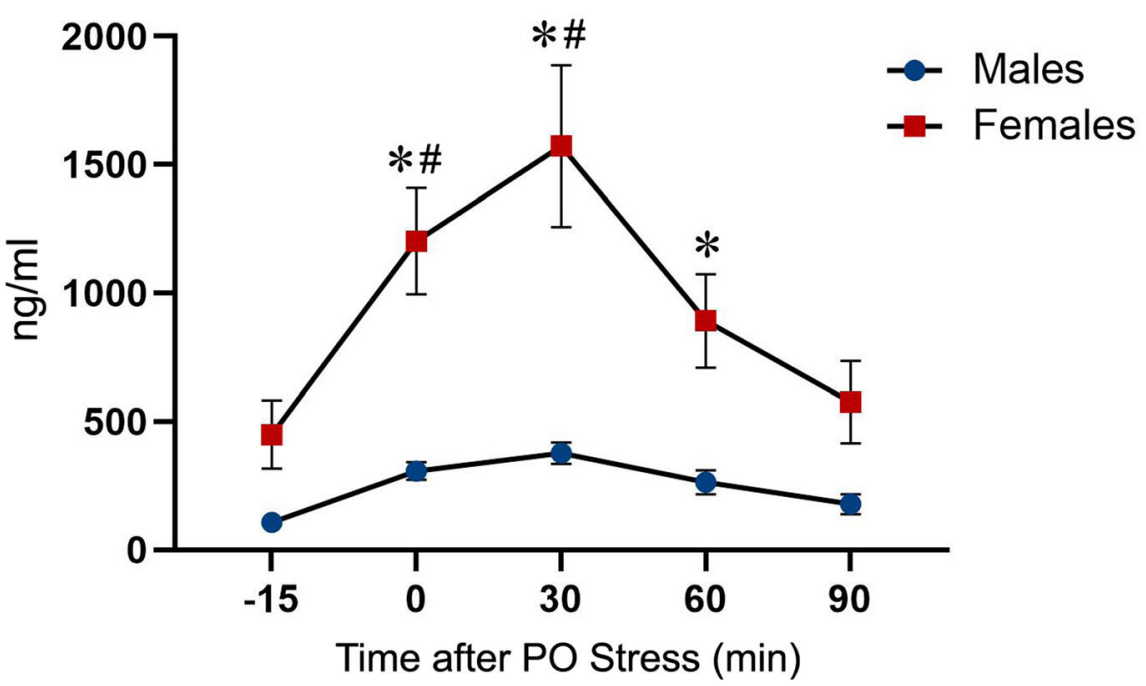

Fig. 3 Plasma corticosterone response in male and female rats following exposure to predator odor $(\mathrm{PO})$ stress. a Experimental design. Rats were exposed to bobcat urine in a clean cage. Tail blood samples were collected before exposure to PO, immediately after, $30 \mathrm{~min}, 60 \mathrm{~min}$, and $90 \mathrm{~min}$ post-stress. $\mathbf{b}$ Plasma corticosterone concentrations at the indicated time points. Data presented as mean \pm SEM. Asterisk denotes $P<.05$ between sexes; number sign denotes $P<.05$ in comparison with the baseline $(-15$ min time point)

weighed and given access to 1 bottle of $20 \% \mathrm{v} / \mathrm{v}$ ethanol and 1 bottle of water approximately $3 \mathrm{~h}$ after the start of the dark cycle on Mondays, Wednesdays, and Fridays. After $24 \mathrm{~h}$, the alcohol bottle was replaced with a second water bottle that was available for the next $24 \mathrm{~h}$. Over the weekends, rats had unlimited access to 2 water bottles after the alcohol bottle was removed on Saturday. Bottles were weighed $24 \mathrm{~h}$ after alcohol presentation. The position of the alcohol bottle was alternated across sessions to control for side preferences. At the last drinking session, tail blood was collected $2 \mathrm{~h}$ after the start of alcohol access to determine blood alcohol concentration (BAC). Rats were restrained manually without anesthesia on a clean surface, and a cut was made $1 \mathrm{~mm}$ from the tip of the tail using a razor blade [34]. Blood samples were collected in $1.5 \mathrm{ml}$ microtube and centrifuged at $1900 \times g$ for $14 \mathrm{~min}$, after which plasma was extracted and immediately analyzed using an Analox AM1 analyzer (Analox Instruments). Alcohol-naive rats (experiment 1 ) were submitted to the same blood collection procedure $24 \mathrm{~h}$ before starting the 5-day conditioned place aversion procedure.

\section{Acoustic startle response test}

At day 2 after exposure to PO stress, rats were tested for acoustic startle reactivity. Rats were placed in a Plexiglas tube attached to an accelerometer inside a dark, soundproof chamber (SR-Lab, San Diego Instruments, CA) and allowed to acclimate for $5 \mathrm{~min}$ (75- $\mathrm{dB}$ background noise) before the test session [35]. This background white noise was present throughout the session. The chamber and Plexiglas tube were cleaned with Quatricide between each animal. Before testing, an S-R calibrator tube was used to calibrate the chambers. The test session consisted of 30 trials with startle stimuli of three different decibel levels: a 750-ms burst of $95 \mathrm{~dB}, 105 \mathrm{~dB}$, or $115 \mathrm{~dB}$ white noise was randomly presented 10 times each, separated by a 30-s fixed inter-trial interval. The maximum startle response (Vmax, arbitrary units) was recorded during the first $100 \mathrm{~ms}$ of each trial. 


\section{Elevated plus-maze test}

The EPM test was used to test locomotor and anxietylike behavior at day 17 after exposure to bobcat urine. The EPM was a black Plexiglas apparatus consisting of two closed arms $(50 \mathrm{~cm} \times 10 \mathrm{~cm} \times 40 \mathrm{~cm})$ and two open arms $(50 \mathrm{~cm} \times 10 \mathrm{~cm})$ attached to metal legs elevating the maze $50 \mathrm{~cm}$ above the ground. All testing was conducted under dim illumination (approximately $10 \mathrm{~lx}$ in the open arms). Rats were placed individually in the center of the maze facing a closed arm and allowed 5 min of free exploration. Behavior was recorded with a video camera positioned above the maze. The EPM was cleaned thoroughly between subjects using Quatricide. Video scoring was done by an observer blind to the conditions; we measured percent time spent in the open arms ((time open/time open + time closed $) \times 100)$, percent entries in the open arms ((entries open/entries open + entries closed) $\times 100$ ), and number of closed arm entries. One arm entry was defined as all four paws entering the arm.

\section{Plasma corticosterone response}

Rats were individually transferred from the homecage to a clean cage in a separate room and exposed to bobcat urine for $15 \mathrm{~min}$. Bobcat urine $(3 \mathrm{ml})$ was added to a sponge that was placed beside the cage. Rats were returned to the homecage after PO exposure. Tail blood was collected in EDTA-covered tubes, as described earlier for BAC, at 5 time points: before exposure to PO, immediately after exposure, $30 \mathrm{~min}, 60 \mathrm{~min}$, and $90 \mathrm{~min}$ post-stress. Samples were centrifuged at $1900 \times g$ for 20 min. Plasma was stored at $-80{ }^{\circ} \mathrm{C}$ and analyzed in duplicate for total corticosterone levels using a DetectX ELISA kit (Arbor Assays, Ann Arbor, MI) according to the manufacturer's instructions.

\section{Statistical analysis}

Data are reported as mean \pm SEM, except where otherwise indicated. All statistics were run using Prism 8 (GraphPad, La Jolla, CA). Alcohol-naive (experiment 1) and alcohol-drinking animals (experiment 2) were not tested in parallel; therefore, they are not analyzed together. Measures of Vmax were normalized by body weight in kilograms. Two-way analysis of variance (ANOVA) was performed to analyze change in time spent in PO-paired chamber, body weight gain, activity in the EPM (percent time in the open arms, percent entries in the open arms, and closed arms entries), and startle reactivity at each decibel level-the variables in all cases were sex and stress condition. Dose of alcohol intake (grams of ethanol per kilogram of body weight per rat, estimated from the pair of rats) is presented as the daily average across 3 measurement days per week. Alcohol consumption and plasma corticosterone were analyzed with two-way repeated measure ANOVAs-the variables were sex and sessions. Fisher's exact test was used to analyze the proportion of avoiders and nonavoiders in each sex. Student's unpaired $t$ test was used to compare the magnitude of avoidance in male and female avoiders. A priori Student's unpaired $t$ tests were used to compare the startle response at each decibel level and activity in the EPM (percent time in the open arms, percent entries in the open arms and closed arms entries) between avoiders and non-avoiders in each sex to determine whether they could be collapsed for subsequent analysis. In cases of significant ANOVA effects, post hoc comparisons were performed using Tukey's multiple comparisons test. Values of $P<.05$ were considered statistically significant.

\section{Results}

Experiment 1: predator odor stress reactivity in alcoholnaive rats

The timeline of experiment 1 procedure is shown in Fig. 1a. Independent of sex, avoiders exhibited significantly greater avoidance of the PO-paired chamber at $24 \mathrm{~h}$ post-exposure $(F(1,44)=78.51, P<.0001)$ relative to non-avoiders (Fig. 1b). There was no significant difference in the magnitude of avoidance between male and female avoiders (Fig. 1b; $t=1.86, P=.08$ ), and the proportion of animals that met the avoider criteria was similar in both sexes (Fig. 1c; $P>.05$ ). Likewise, both male and female rats exposed to PO exhibited significantly reduced body weight gain relative to unstressed controls $24 \mathrm{~h}$ post-stress (Fig. $1 \mathrm{~d}$; stress effect: $F(1,44)$ $=6.99, P=.01$ ) but not 4 days post-stress (stress effect: $F(1,44)=1.12, P=.29$; data not shown).

Because we did not find significant differences in the ASR between avoider and non-avoider males ( $95 \mathrm{~dB}: t=$ .61, $P=.54 ; 105 \mathrm{~dB}: t=.20, P=.84 ; 115 \mathrm{~dB}: t=.92, P=$ .36) nor between avoider and non-avoider females $(95$ $\mathrm{dB}: t=.59, P=.56 ; 105 \mathrm{~dB}: t=.45, P=.65 ; 115 \mathrm{~dB}: t=$ $.55, P=.59$ ), animals exposed to bobcat urine were pooled into a group designated PO stress and compared to unstressed controls, segregated by sex. To determine whether PO stress affected startle reactivity differently in male and female rats, ASR data were analyzed for each decibel. At $95 \mathrm{~dB}$ (Fig. 1e), we found a significant main effect of $\operatorname{sex}(F(1,58)=5.45, P=.02)$ and a sex $\times$ stress interaction effect $(F(1,58)=10.59, P=.002)$. Tukey's post hoc comparisons revealed that control females exhibited higher startle reactivity than control males $(P=$ $.01)$ and that PO stressed females showed lower startle reactivity relative to control females $(P=.01)$. At $105 \mathrm{~dB}$ (Fig. 1f), we found a significant sex $\times$ stress interaction effect $(F(1,58)=4.11, P=.047)$. Tukey's post hoc comparison revealed that $\mathrm{PO}$ stressed females exhibited lower startle reactivity than control females $(P=.02)$. At 
$115 \mathrm{~dB}$ (Fig. 1g), we found a significant sex $\times$ stress interaction effect $(F(1,58)=6.02, P=.02)$. Tukey's post hoc comparison revealed that PO stressed males exhibited higher startle reactivity than PO stressed females $(P$ $=.0008$ ).

At day 17 post-stress, rats were tested for locomotor activity and anxiety-like behavior in the EPM. Again, we did not find significant differences between avoider and non-avoider males nor between avoider and non-avoider females on percent time in the open arms (males: $t=$ 1.58, $P=.13$; females: $t=1.25, P=.22$ ), percent entries in the open arms (males: $t=1.38, P=.18$; females: $t=$ .87, $P=.39$ ), and closed arms entries (males: $t=.80, P=$ .43; females: $t=1.06, P=.30$ ); thus, animals exposed to bobcat urine were pooled into a group designated PO stress and compared to unstressed controls, segregated by sex. There was no significant effect of sex $(F(1,59)=$ $.73, P=.39)$ or stress $(F(1,59)=.04, P=.84)$ on percent time in the open arms of the EPM (Table 1). Similarly, we did not find significant effect of $\operatorname{sex}(F(1,59)=.08$, $P=.78)$ or stress $(F(1,59)=.009, P=.92)$ on percent entries in the open arms of the EPM (Table 1). General locomotor performance was assessed by counting closed arm entries in the EPM. Neither sex $(F(1,59)=.59, P=$ $.45)$ nor stress $(F(1,59)=3.65, P=.06)$ affected the number of closed arms entries (Table 1 ).

\section{Experiment 2: predator odor stress reactivity in rats with a history of alcohol drinking}

Timeline of experiment 2 procedure is shown in Fig. 2a. Before exposure to PO stress, pair-housed rats were given intermittent access to alcohol for 5 weeks. There was no significant difference on alcohol consumption over the weeks between male and female rats (Fig. 2d; $F$ $(1,15)=1.29, P=.27)$. Likewise, we did not find sex difference on BACs analyzed $2 \mathrm{~h}$ after the beginning of the last drinking session (Fig. 2d; $t=.21, P=.83$ ). Independent of sex, avoiders exhibited significantly greater avoidance of the PO-paired chamber at $24 \mathrm{~h}$ post-exposure ( $F$ $(1,18)=28.79, P<.0001)$ relative to non-avoiders (Fig. $2 b)$. There was no significant difference in the magnitude of avoidance between male and female avoiders (Fig. $2 \mathrm{~b} ; t=.76, P=.48$ ). Although the difference in the proportion of avoiders and non-avoiders in male and

Table 1 Elevated plus-maze activity in alcohol-naive male and female rats at day 17 after exposure to predator odor (PO) stress

\begin{tabular}{llllll}
\hline & \multicolumn{3}{l}{ Males } & & Females \\
\cline { 2 - 3 } & Control & PO stress & & Control & PO stress \\
\hline \% time open arms & $29.1 \pm 8.0$ & $19.5 \pm 3.3$ & & $16.5 \pm 6.4$ & $24.2 \pm 2.9$ \\
\% entries open arms & $30.4 \pm 8.1$ & $23.1 \pm 3.3$ & & $21.3 \pm 7.3$ & $29.5 \pm 2.7$ \\
Closed arms entries & $10.9 \pm 1.0$ & $12.2 \pm 0.7$ & & $9.3 \pm 2.2$ & $12.1 \pm 0.7$ \\
\hline
\end{tabular}

Data presented as mean \pm SEM female rats did not reach statistical significance $(P=.39)$ due to sample size (given a 95\% confidence level and $80 \%$ power, the recommended sample size would be $n=$ 95), the proportion of alcohol-drinking males that met the avoider criteria was $27 \%$ higher than alcoholdrinking females (Fig. 2c).

Because we did not find significant differences in the ASR between avoider and non-avoider males $(95 \mathrm{~dB}: t=$ 1.06, $P=.32$; $105 \mathrm{~dB}: t=.50, P=.63 ; 115 \mathrm{~dB}: t=.77, P=$ .46) nor between avoider and non-avoider females $(95$ $\mathrm{dB}: t=.72, P=.49 ; 105 \mathrm{~dB}: t=.42, P=.68 ; 115 \mathrm{~dB}: t=$ $.06, P=.95)$, animals exposed to bobcat urine were pooled into a group designated PO stress and compared to unstressed controls, segregated by sex. To determine whether PO stress affected startle reactivity differently in male and female rats with a history of alcohol drinking, ASR data were analyzed for each decibel. At $95 \mathrm{~dB}$ (Fig. 2e), we found a significant sex $\times$ stress interaction effect $(F(1,29)=7.02, P=.01)$. Tukey's post hoc comparison revealed that $\mathrm{PO}$ stressed males exhibited higher startle reactivity than control males $(P=.03$ ). At $105 \mathrm{~dB}$ (Fig. 2f), we found a significant sex $\times$ stress interaction effect $(F(1,29)=5.94, P=.02)$. Tukey's post hoc comparisons revealed that $\mathrm{PO}$ stressed males exhibited higher startle reactivity relative to control males $(P=.04)$ and also relative to PO stressed females $(P=.004)$. At $115 \mathrm{~dB}$ (Fig. 2g), we found a significant main effect of sex $(F(1$, 29) $=5.23, P=.03)$ on ASR.

At day 17 post-stress, rats were tested for locomotor activity and anxiety-like behavior in the EPM. We did not find significant differences between avoider and non-avoider males nor between avoider and non-avoider females on percent time in the open arms (males: $t=$ $.23, P=.82$; females: $t=.63, P=.55$ ), percent entries in the open arms (males: $t=.44, P=.67$; females: $t=.50, P$ $=.63$ ), and closed arm entries (males: $t=.23, P=.82$; females: $t=.62, P=.55$ ); thus, animals exposed to bobcat urine were pooled into a group designated PO stress and compared to unstressed controls, segregated by sex. Regardless of stress condition, females with a history of alcohol drinking spent more time in the open arms of the

Table 2 Elevated plus-maze activity in alcohol-drinking male and female rats at day 17 after exposure to predator odor (PO) stress

\begin{tabular}{llllll}
\hline & Males & & & \multicolumn{2}{l}{ Females* } \\
\cline { 2 - 3 } & Control & PO stress & & Control & PO stress \\
\hline \% time open arms & $12.5 \pm 6.9$ & $13.9 \pm 3.1$ & & $22.0 \pm 6.2$ & $39.2 \pm 5.7$ \\
\% entries open arms & $17.5 \pm 8.4$ & $21.0 \pm 4.4$ & & $31.1 \pm 6.7$ & $38.3 \pm 1.7$ \\
Closed arms entries & $10.0 \pm 1.1$ & $10.9 \pm 0.8$ & & $12.5 \pm 1.5$ & $12.6 \pm 0.7$ \\
\hline
\end{tabular}

Data presented as mean \pm SEM. Regardless of stress condition, females spent more time in the open arms and made more entries into the open and closed arms of the EPM relative to males ${ }^{*} P<.05$ 
EPM relative to males $($ Table 2; $F(1,30)=11.86, P=$ .002). Similarly, alcohol-drinking females made more entries in the open arms (Table 2; $F(1,30)=10.17, P=$ $.003)$ and in the closed arm of the EPM relative to males (Table 2; $F(1,30)=4.96, P=.03)$.

\section{Experiment 3: predator odor stress effects on plasma corticosterone response}

The timeline of experiment 3 procedure is shown in Fig. 3a. PO stress produced sexually dimorphic changes in circulating corticosterone levels (Fig. 3b). A two-way repeated measure ANOVA yielded a significant main effect of sex $(F(1,9)=27.21, P=.0006)$, time $(F(4,34)=$ 11.83, $P<.0001)$, and a sex $\times$ time interaction effect $(F$ $(4,34)=4.65, P=.004)$ on plasma corticosterone response following exposure to bobcat urine. Tukey's post hoc comparison revealed that corticosterone levels were significantly higher in females relative to males immediately after PO exposure $(P=.0002), 30 \mathrm{~min}(P<.0001)$, and $60 \mathrm{~min}$ post-stress $(P=.012)$. Moreover, in females, corticosterone levels immediately after PO $(P=.0005)$ and $30 \mathrm{~min}$ post-stress $(P<.0001)$ were higher than the baseline.

\section{Discussion}

We report that alcohol-naive male and female rats exposed to PO displayed blunted weight gain $24 \mathrm{~h}$ poststress even though only a subset of stressed animals exhibited avoidance behavior. A similar percentage of alcohol-naive males and females were classified as avoiders after stress, but the proportion of avoiders was higher in alcohol-experienced males relative to females with a history of alcohol drinking. Moreover, PO exposure enhanced startle reactivity in alcohol-experienced males, and females exhibited blunted startle reactivity following odor exposure, regardless of alcohol drinking history. Alcohol-experienced females presented lower anxiety-like behavior and higher general activity in the EPM relative to alcohol-experienced males. Finally, bobcat urine exposure significantly increased circulating corticosterone levels in females immediately after stress until $60 \mathrm{~min}$ post-stress compared with males.

In the present study, pair-housed male and female rats consumed comparable amounts of alcohol and achieved similar BACs $2 \mathrm{~h}$ after the last drinking session. Thus, sex differences in post-stress startle reactivity are likely not attributable to different levels of alcohol consumption. It is worth mentioning that our animals were pairhoused throughout the experiment, whereas in most studies using the 2-bottle choice protocol, rats were housed individually $[33,36]$. While our housing condition does not allow direct associations between alcohol intake and behavioral outcomes in individual animals, it has the benefit of avoiding potentially confounding stressful effects of social isolation.

In line with previous studies $[27,37,38]$, bobcat urine exposure elicited significant avoidance in a subset of animals. A similar proportion of alcohol-naive male and female rats exposed to PO were classified as avoiders (males 42\%; females 37\%), with similar magnitude of bobcat urine-paired context avoidance $24 \mathrm{~h}$ post-stress. In animals with a history of ethanol consumption, a higher percentage of males was classified as avoiders (males 67\%; females 40\%). Interestingly, a systematic review of the comorbidity between PTSD and alcohol misuse found associations between alcohol consumption and the avoidance/numbing and hyperarousal PTSD symptom clusters [39]. Moreover, our group demonstrated that male avoider rats exhibit persistent increases in alcohol self-administration, and avoidance behavior predicts post-stress escalation of alcohol drinking [27, 38].

Although only a subset of rats exposed to PO displayed avoidance behavior, all stressed animals exhibited signs of physiological stress. Our laboratory has previously reported that both avoider and non-avoider male rats exhibited increased anxiety-like behavior following exposure to PO [40]. The current data build on that work by showing that all alcohol-naive stressed male and female rats displayed blunted weight gain $24 \mathrm{~h}$ after PO exposure (we did not evaluate changes in body weight in rats with a history of alcohol drinking). Additionally, male rats exposed to bobcat urine have been shown to exhibit a non-significant general increase in startle reactivity [35]. We confirmed and extended these findings by including females and testing animals with a history of chronic alcohol drinking. Here, when tested 6 days after the last drinking session, only alcoholexperienced males showed stress-induced increase in startle reactivity following bobcat urine exposure.

PO stress reduced startle reactivity in females, regardless of alcohol drinking history. Even though we did not measure baseline ASR to counterbalance the groups before the test session, it is unlikely that random assignment of animals would select for rats with different startle reactivity. Prior work has reported lower startle reactivity in female rats after inescapable tail shock stress and identified the presence of ovarian hormones as an important element of stress-induced startle suppression [41]. Interestingly, startle suppression was demonstrated to be more evident when tail shock was applied during estrus [42]. Contrary to these findings, there was no effect of the estrous cycle on adrenocorticotropic hormone (ACTH) and corticosterone responses in female rats exposed to acute restraint stress [43]. In the present study, we did not investigate the effects of estrous cycle on stress reactivity; therefore, the influence of ovarian 
hormones on behavioral and endocrine responses associated with PO exposure is still to be determined.

Our group reported that bobcat urine exposure increased anxiety-like behavior in male rats 2 and 5 days post-stress [40]. Here, we show that bobcat urine exposure did not alter anxiety-like behavior at day 17 poststress in alcohol-naive rats. In animals with a history of alcohol drinking, females exhibited lower anxiety-like behavior than males in the EPM (i.e., increased open arm time and open arm entries), and also higher spontaneous motor activity (i.e., increased closed arm entries). Therefore, we cannot rule out the possibility that changes in open arm exploration were affected by alterations in general activity. Similar increase in exploratory activity levels on the EPM was described in Long-Evans female rats following 6-week intermittent access to $20 \%$ ethanol in comparison with males exposed to the same procedure [44]. Thus, the higher activity in the EPM observed in females may reflect sex differences in stress/ fear coping strategies rather than reduced anxiety-like behavior. In fact, a study using factor analysis showed that in females, activity, rather than anxiety, emerged as the strongest factor in the EPM [45]. Likewise, females are four times more likely than males to display fear in the form of rapid movements instead of freezing in traditional models of Pavlovian fear conditioning [46].

Corticosterone response was higher in females than males immediately after exposure to PO stress until 60 min post-stress, when it returned to baseline levels. Our group showed that male avoider rats exhibit attenuated corticosterone response immediately following exposure to bobcat urine [40]. Differently from this study, animals used here to evaluate sex differences in the time course of corticosterone response were exposed to bobcat urine in a clean cage instead of in the 3-chamber apparatus; thus, they were not indexed as avoiders or non-avoiders. In line with our findings, female rats exposed to acute restraint stress exhibit higher ACTH and corticosterone responses than males, in addition to significantly higher c-fos mRNA expression in the paraventricular nucleus of the hypothalamus [43]. Conversely, TMT exposure elicits similar increase in circulating corticosterone in both male and female Wistar rats [47], indicating that distinct POs (i.e., natural olfactory stimuli or synthetic olfactory stimulus) can produce different responses. Notably, suppression of ASR following combined cat exposure and saline injection in male rats can be blocked by substituting the injected saline with a glucocorticoid receptor antagonist [48].

Systemically circulating corticosterone is coupled to different carriers, mainly corticosteroid binding globulin (CBG) [49]. These proteins play a critical role in regulating glucocorticoid bioavailability by sequestering it in an inactive complex $[50,51]$. In mature female rats, CBG content in the serum is 2.5 times higher than in agematched males [52] because of estradiol-induced increases in hepatic CBG synthesis [53]. This finding highlights the need for future studies aimed at evaluating sex differences in stress reactivity to test levels of bioavailable free corticosterone to determine the biological significance of altered corticosterone levels.

\section{Conclusions}

We report robust sex differences in behavioral and endocrine responses to bobcat urine exposure in adult Wistar rats. Males and females exposed to PO displayed blunted weight gain $24 \mathrm{~h}$ post-stress, but only a subset of stressed animals exhibited avoidance behavior. Male rats with a history of chronic moderate alcohol drinking exhibited increased traumatic stress reactivity relative to alcohol-experienced females. Regardless of alcohol drinking history, stressed females exhibited blunted startle reactivity compared with unstressed control females and stressed males. Finally, females presented enhanced corticosterone response following PO stress.

\section{Perspectives and significance}

Sex differences in traumatic stress responses are among the most widely reported phenomena in epidemiological and clinical studies. To the best of our knowledge, the findings reported here are the first to provide evidence that a history of chronic moderate alcohol drinking differentially modulates PO stress reactivity in male and female rats. Our data support the notion that females rather respond differently to trauma and open doors for future work aimed at testing the neurobiology underlying sex differences in traumatic stress reactivity.

\section{Acknowledgements}

This study was supported by the National Institutes of Health grants R01AA023305, R01AA026531 and the Cohen Veterans Bioscience Foundation grant PJ-011-103. This work was also supported in part by Merit Review Award \#101 BX003451 (NWG) from the United States (U.S.) Department of Veterans Affairs, Biomedical Laboratory Research and Development Service. The authors thank Curtis Vande Stouwe for his technical support and Tivon M. Eugene for his assistance in data collection.

\section{Authors' contributions}

LA-S conceived and planned the study, performed the experiments, analyzed data, and wrote the paper. CLS performed the experiments. NWG conceived and planned the study, supervised the project, and contributed to the final version of the manuscript. All authors read and approved the final manuscript.

\section{Funding}

This study was supported by the National Institutes of Health grants R01AA023305 and R01AA026531; by Cohen Veterans Bioscience grant PJ011-103; and by U.S. Department of Veterans Affairs grant I01BX003451.

Availability of data and materials

All data are available from the corresponding author upon request. 


\section{Ethics approval}

All procedures were approved by the Institutional Animal Care and Use Committee of the Louisiana State University Health Sciences Center and were in accordance with the National Institute of Health Guidelines.

\section{Consent for publication}

Not applicable.

\section{Competing interests}

NWG owns shares in Glauser Life Sciences, a company with interest in developing therapeutics for mental health disorders. There is no direct link between those interests and the work contained herein.

\section{Author details}

${ }^{1}$ Department of Physiology, School of Medicine, Louisiana State University Health Sciences Center, New Orleans, LA, USA. ${ }^{2}$ Alcohol \& Drug Center of Excellence, School of Medicine, Louisiana State University Health Sciences Center, New Orleans, LA, USA. ${ }^{3}$ Neuroscience Center of Excellence, School of Medicine, Louisiana State University Health Sciences Center, New Orleans, LA, USA. ${ }^{4}$ Southeast Louisiana Veterans Health Care System (SLVHCS) New Orleans, LA, USA.

\section{Received: 27 November 2019 Accepted: 24 April 2020}

\section{Published online: 11 May 2020}

\section{References}

1. American Psychiatric Association. Diagnostic and statistical manual of mental disorders. 5th ed. Arlington: American Psychiatric Publishing; 2013.

2. Breslau N, Peterson EL, Schultz LR. A second look at prior trauma and the posttraumatic stress disorder effects of subsequent trauma: a prospective epidemiological study. Arch Gen Psychiatry. 2008;65:431-7.

3. Kessler RC, Sonnega A, Bromet E, Hughes M, Nelson CB. Posttraumatic stress disorder in the national comorbidity survey. Arch Gen Psychiatry. 1995;52: 1048-60.

4. Miles SR, Menefee DS, Wanner J, Tharp AT, Kent TA. The relationship between emotion dysregulation and impulsive aggression in veterans with posttraumatic stress disorder symptoms. J Interpers Violence. 2016;31:1795816.

5. Vujanovic AA, Rathnayaka N, Amador CD, Schmitz JM. Distress tolerance: associations with posttraumatic stress disorder symptoms among traumaexposed, cocaine-dependent adults. Behav Modif. 2016;40:1-24.

6. Gilpin NW, Weiner JL. Neurobiology of comorbid post-traumatic stress disorder and alcohol-use disorder. Genes Brain Behav. 2017:16:15-43.

7. Kessler RC, Chiu WT, Demler O, Merikangas KR, Walters EE. Prevalence, severity, and comorbidity of 12-month DSM-IV disorders in the National Comorbidity Survey Replication. Arch Gen Psychiatry. 2005;62:617-27.

8. Blanco C, Xu Y, Brady K, Pérez-Fuentes G, Okuda M, Wang S. Comorbidity of posttraumatic stress disorder with alcohol dependence among U.S. adults: results from National Epidemiological Survey on Alcohol and Related Conditions. Drug Alcohol Depend. 2013;132:630-8.

9. Seal KH, Cohen G, Waldrop A, Cohen BE, Maguen S, Ren L. Substance use disorders in Iraq and Afghanistan veterans in VA healthcare, 2001-2010: implications for screening, diagnosis and treatment. Drug Alcohol Depend. 2011:116:93-101.

10. Smith NDL, Cottler LB. The epidemiology of post-traumatic stress disorder and alcohol use disorder. Alcohol Res. 2018;39:113-20.

11. Able ML, Benedek DM. Severity and symptom trajectory in combat-related PTSD: a review of the literature. Curr Psychiatry Rep. 2019:21:58.

12. Stanley $I H$, Rogers ML, Hanson JE, Gutierrez PM, Joiner TE. PTSD symptom clusters and suicide attempts among high-risk military service members: a three-month prospective investigation. J Consult Clin Psychol. 2019;87:6778.

13. Shalev AY, Orr SP, Peri T, Schreiber S, Pitman RK. Physiologic responses to loud tones in Israeli patients with posttraumatic stress disorder. Arch Gen Psychiatry. 1992:49:870-5.

14. Shalev AY, Peri T, Orr SP, Bonne O, Pitman RK. Auditory startle responses in help-seeking trauma survivors. Psychiatry Res. 1997:69:1-7.

15. Shalev AY, Peri T, Brandes D, Freedman S, Orr SP, Pitman RK. Auditory startle response in trauma survivors with posttraumatic stress disorder: a prospective study. Am J Psychiatry. 2000;157:255-61.
16. Morgan CA 3rd, Grillon C, Southwick SM, Davis M, Charney DS. Exaggerated acoustic startle reflex in Gulf War veterans with posttraumatic stress disorder. Am J Psychiatry. 1996;153:64-8.

17. Grillon C, Morgan CA, Southwick SM, Davis M, Charney DS. Baseline startle amplitude and prepulse inhibition in Vietnam veterans with posttraumatic stress disorder. Psychiatry Res. 1996;64:169-78.

18. Grillon C, Morgan CA 3rd, Davis M, Southwick SM. Effects of experimental context and explicit threat cues on acoustic startle in Vietnam veterans with posttraumatic stress disorder. Biol Psychiatry. 1998;44:1027-36.

19. Medina AM, Mejia WY, Schell AM, Dawson ME, Margolin G. Startle reactivity and PTSD symptoms in a community sample of women. Psychiatry Res. 2001;101:157-69.

20. Ornitz EM, Pynoos RS. Startle modulation in children with posttraumatic stress disorder. Am J Psychiatry. 1989;146:866-70.

21. Lebron-Milad K, Milad MR. Sex differences, gonadal hormones and the fear extinction network: implications for anxiety disorders. Biol Mood Anxiety Disord. 2012:2:3

22. Shansky RM. Sex differences in PTSD resilience and susceptibility: challenges for animal models of fear learning. Neurobiol Stress. 2015;1:60-5.

23. Cohen $\mathrm{H}$, Zohar J, Matar M. The relevance of differential response to trauma in an animal model of posttraumatic stress disorder. Biol Psychiatry. 2003:53:463-73.

24. Cohen H, Matar MA, Richter-Levin G, Zohar J. The contribution of an animal model toward uncovering biological risk factors for PTSD. Ann N Y Acad Sci. 2006;1071:335-50

25. Corley MJ, Caruso MJ, Takahashi LK. Stress-induced enhancement of fear conditioning and sensitization facilitates extinction-resistant and habituation resistant fear behaviors in a novel animal model of posttraumatic stress disorder. Physiol Behav. 2012;105:408-16.

26. Kondoh $K$, Lu Z, Ye X, Olson DP, Lowell BB, Buck LB. A specific area of olfactory cortex involved in stress hormone responses to predator odours. Nature. 2016;532:103-6.

27. Edwards S, Baynes BB, Carmichael CY, Zamora-Martinez ER, Barrus M, Koob GF, Gilpin NW. Traumatic stress reactivity promotes excessive alcohol drinking and alters the balance of prefrontal cortex-amygdala activity. Transl Psychiatry. 2013;3:e296.

28. Takahashi LK, Nakashima BR, Hong H, Watanabe K. The smell of danger: a behavioral and neural analysis of predator odor-induced fear. Neurosci Biobehav Rev. 2005:29:1157-67.

29. Endres T, Apfelbach R, Fendt M. Behavioral changes induced in rats by exposure to trimethylthiazoline, a component of fox odor. Behav Neurosci. 2005;119:1004-10.

30. Mackenzie L, Nalivaiko E, Beig MI, Day TA, Walker FR. Ability of predator odour exposure to elicit conditioned versus sensitised post traumatic stress disorder-like behaviours, and forebrain deltaFosB expression, in rats. Neuroscience. 2010;169:733-42.

31. Albrechet-Souza L, Gilpin NW. The predator odor avoidance model of posttraumatic stress disorder in rats. Behav Pharmacol. 2019;30:105-14.

32. Ferrero DM, Lemon JK, Fluegge D, Pashkovski SL, Korzan WJ, Datta SR, Spehr M, Fendt M, Liberles SD. Detection and avoidance of a carnivore odor by prey. Proc Natl Acad Sci U S A. 2011;108:11235-40.

33. Simms JA, Steensland P, Medina B, Abernathy KE, Chandler LJ, Wise R, Bartlett SE. Intermittent access to $20 \%$ ethanol induces high ethano consumption in Long-Evans and Wistar rats. Alcohol Clin Exp Res. 2008;32: 1816-23.

34. Avegno EM, Gilpin NW. Inducing alcohol dependence in rats using chronic intermittent exposure to alcohol vapor. Bio Protoc. 2019;9:e3222.

35. Roltsch EA, Baynes BB, Mayeux JP, Whitaker AM, Baiamonte BA, Gilpin NW. Predator odor stress alters corticotropin-releasing factor-1 receptor (CRF1R)dependent behaviors in rats. Neuropharmacology. 2014;79:83-9.

36. Carnicella S, Ron D, Barak S. Intermittent ethanol access schedule in rats as a preclinical model of alcohol abuse. Alcohol. 2014;48:243-52.

37. Schreiber AL, Lu YL, Baynes BB, Richardson HN, Gilpin NW. Corticotropinreleasing factor in ventromedial prefrontal cortex mediates avoidance of a traumatic stress-paired context. Neuropharmacology. 2017:113:323-30.

38. Weera MM, Schreiber AL, Avegno EM, Gilpin NW. The role of central amygdala corticotropin-releasing factor in predator odor stress-induced avoidance behavior and escalated alcohol drinking in rats. Neuropharmacology. 2020;166:107979.

39. Debell F, Fear NT, Head M, Batt-Rawden S, Greenberg N, Wessely S, Goodwin L. A systematic review of the comorbidity between PTSD and alcohol misuse. Soc Psychiatry Psychiatr Epidemiol. 2014;49:1401-25. 
40. Whitaker AM, Gilpin NW. Blunted hypothalamo-pituitary adrenal axis response to predator odor predicts high stress reactivity. Physiol Behav. 2015;147:16-22.

41. Beck KD, Servatius RJ. Stress-induced reductions of sensory reactivity in female rats depend on ovarian hormones and the application of a painful stressor. Horm Behav. 2005:47:532-9.

42. Beck KD, Jiao X, Cominski TP, Servatius RJ. Estrus cycle stage modifies the presentation of stress-induced startle suppression in female Sprague-Dawley rats. Physiol Behav. 2008;93:1019-23.

43. Babb JA, Masini CV, Day HE, Campeau S. Sex differences in activated corticotropin-releasing factor neurons within stress-related neurocircuitry and hypothalamic-pituitary-adrenocortical axis hormones following restraint in rats. Neuroscience. 2013;234:40-52.

44. Nelson NG, Suhaidi FA, Law WX, Liang NC. Chronic moderate alcohol drinking alters insulin release without affecting cognitive and emotion-like behaviors in rats. Alcohol. 2018;70:11-22.

45. Fernandes C, González MI, Wilson CA, File SE. Factor analysis shows that female rat behaviour is characterized primarily by activity, male rats are driven by sex and anxiety. Pharmacol Biochem Behav. 1999;64:731-8.

46. Gruene TM, Flick K, Stefano A, Shea SD, Shansky RM. Sexually divergent expression of active and passive conditioned fear responses in rats. Elife. 2015:4.

47. Homiack D, O'Cinneide E, Hajmurad S, Barrileaux B, Stanley M, Kreutz MR, Schrader LA. Predator odor evokes sex-independent stress responses in male and female Wistar rats and reduces phosphorylation of cyclicadenosine monophosphate response element binding protein in the male, but not the female hippocampus. Hippocampus. 2017;27:1016-29.

48. Adamec R, Strasser K, Blundell J, Burton P, McKay DW. Protein synthesis and the mechanisms of lasting change in anxiety induced by severe stress. Behav Brain Res. 2006;167:270-86.

49. Hammond GL. Potential functions of plasma steroid-binding proteins. Trends Endocrinol Metab. 1995;6:298-304.

50. Kudielka BM, Kirschbaum C. Sex differences in HPA axis responses to stress: a review. Biol Psychol. 2005;69:113-32.

51. Perogamvros I, Ray DW, Trainer PJ. Regulation of cortisol bioavailability-effects on hormone measurement and action. Nat Rev Endocrinol. 2012;8: 717-27.

52. Mataradze GD, Kurabekova RM, Rozen VB. The role of sex steroids in the formation of sex-differentiated concentrations of corticosteroid-binding globulin in rats. J Endocrinol. 1992;132:235-40.

53. Feldman D, Mondon CE, Horner JA, Weiser JN. Glucocorticoid and estrogen regulation of corticosteroid-binding globulin production by rat liver. Am J Phys. 1979;237:E493-9.

\section{Publisher's Note}

Springer Nature remains neutral with regard to jurisdictional claims in published maps and institutional affiliations.

Ready to submit your research? Choose BMC and benefit from:

- fast, convenient online submission

- thorough peer review by experienced researchers in your field

- rapid publication on acceptance

- support for research data, including large and complex data types

- gold Open Access which fosters wider collaboration and increased citations

- maximum visibility for your research: over $100 \mathrm{M}$ website views per year

At $\mathrm{BMC}$, research is always in progress.

Learn more biomedcentral.com/submissions 